\section{American Society of Clinical Oncology highlights 2013: breast cancer and gynecological malignancies}

\section{Nadia Harbeck}

Breast Center, University of Munich (Department OB\&GYN Maistrasse \& GroBhadern), Marchioninistrasse 15, 81377 Munich, Germany = Tel. : +49 8970957581 = Fax: +49 8970957582

=nadia.harbeck@med.uni-muenchen.de

\section{American Society of Clinical Oncology 2013 Annual Meeting}

31 May - 4 June 2013, Chicago, IL, USA

The American Society of Clinical Oncology 2013 Annual Meeting took place from May 31 until June 42013 in Chicago (IL, USA). Highlights within the area of gynecological oncology and breast cancer included two studies in cervical cancer addressing screening as well as treatment of advanced disease. In breast cancer, the presented studies covered a wide range of topics from local therapy to targeted therapy in the advanced setting. They were not practice changing but rather for the most part confirmatory of earlier findings. Last but not least, a positive Phase III study, BOLERO III, proved the hypothesis that trastuzumab resistance can be overcome by intracellular signal transduction using the mTOR inhibitor everolimus.

\section{Gynecological cancers}

A cluster randomized controlled trials evaluating the impact of cervical cancer screening was able to demonstrate that biannual visual inspection with acetic acid screening significantly downstaged cervical cancers ( 50 vs $24 \leq$ stage IIB; $\mathrm{p}=0.002$ ) and decreased cervical cancer mortality (67 vs 90; hazard ratio [HR]: 0.685; $\mathrm{p}=0.003)$. The observed $31 \%$ mortality reduction was estimated by the authors to amount to the prevention of more than 22,000 annual cervical cancer deaths in India and 72,000 worldwide [1].

In advanced primary stage IVB or recurrent/persistent cervical cancer (GOG 240), the nonplatinum doublet topotecan/paclitaxel was not able to improve survival compared with cisplatinum/paclitaxel. However, the addition of bevacizumab $(15 \mathrm{mg} / \mathrm{kg}$ every 3 weeks) significantly improved progressionfree survival (PFS; median 8.2 vs 5.9 months; HR: 0.67; $\mathrm{p}=0.0002$ ) as well as overall survival (OS; median: 17 vs 13.3 months; HR: 0.71; $\mathrm{p}=0.0035)$. There were significant differences favoring the chemotherapy-only arm for gastrointestinal and genitourinary fistulas, hypertension, neutropenia and thromboembolism [2]. Given these mostly numerically low but significant differences in side effects, more extensive data analysis will hopefully be able to elucidate which patients derive particular benefit from the antiangiogenic regimen.
In ovarian cancer patients without progression after first-line chemotherapy, the AGO-OVAR 16 trial evaluated the impact of a 24-month maintainance therapy with pazopanib [3]. The study met its primary end point with pazopanib significantly prolonging PFS (median 17.9 vs 12.3 months; HR: 0.76; $\mathrm{p}=0.0021$ ). After a median observation time of 24 months, only approximately $20 \%$ of the events needed for OS analysis had been observed. There was an increase in several grade 3-4 side effects with pazopanib versus placebo consistent with tyrosine-kinase inhibitor therapy. Given the maintenance data for bevacizumab from ICON 7 [4] and GOG 218 [5], the overall therapeutic ratio (benefit vs side effect) will certainly decide whether, and if so which, maintenance therapy is suitable for an individual ovarian cancer patient after first-line chemotherapy. In particular, considering the potential toxicities, a biomarker-based therapy approach would certainly be preferable.

\section{Early breast cancer}

Two studies in locoregional therapy addressed axilla management: the NSABP and AMAROS trials. Long-term results of NSABP-B32 confirm the modern standard of sentinel-lymph node biopsy in a randomized comparison to axillary lymph node dissection (AD). In 3986 clinically node-negative patients, only 81 (sentinel lymph node biopsy) versus 84 relapses (AD) were
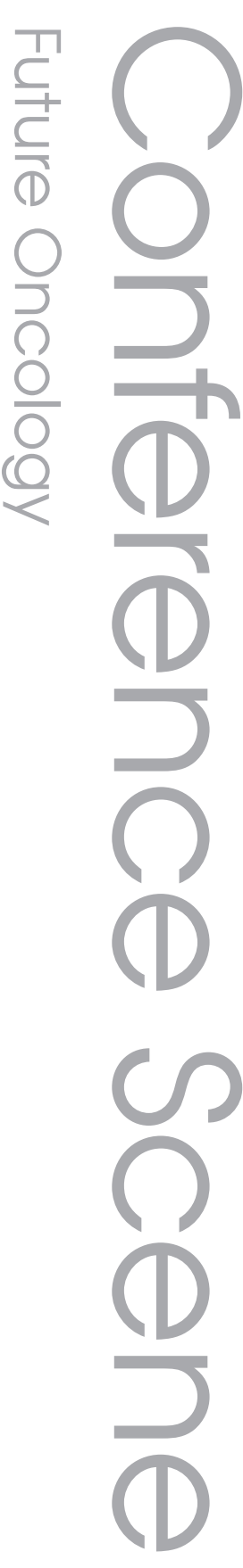

\section{Keywords}

- breast cancer

- gynecological cancer

- targeted therapy Future 
detected (HR: 0.96; $\mathrm{p}=0.77$ ). After 10 years, immunohistochemical detection of occult tumor cells (616 out of 3268) did not have an impact on OS and was, thus, not recommended by the authors [6]. The EORTC AMAROS trial randomized patients with positive sentinel nodes to subsequent $\mathrm{AD}$ versus lymph node radiation. In $67.1 \%$ of patients no further involved lymph nodes were detected after AD. Local relapse rates after 5 years were very low in both arms at 0.43 (AD) and $1.19 \%$ (radiation), respectively, and OS did not differ (HR: 1.17; $\mathrm{p}=0.34$ ). Whereas $\mathrm{AD}$ was associated with more lymph edema even after 5 years (28 vs $13 \%$; p > 0.0001), radiation therapy led to a trend in impaired arm mobility [7]. The authors concluded that radiation treatment could be considered standard in cases of positive sentinel lymph nodes. However, given the recent change of axillary management in forgoing $\mathrm{AD}$ in selected patients with one to two involved sentinel nodes, the collective for whom the AMAROS results can be directly applied to seems rather small.

The question of avoiding overtreatment by adjuvant chemotherapy in luminal tumors was addressed by a common analysis of ABCSG-8 and transATAC tumors using the PAM50 assay. In patients with one to three involved lymph nodes ( $\mathrm{n}=543$; all endocrine responsive), PAM50 was able to identify a low-risk group with a 10 -year metastasis-free survival of less than $10 \%$. In total, $71 \%$ of patients with one lymph node had a luminal A tumor and an $8.4 \%$ 10 -year metastasis-free survival [8]. These data underline the importance of current trial concepts, such as WSG-ADAPT, which tries to spare patients with zero to three lymph nodes from chemotherapy if they have a low or intermediate Oncotype DX ${ }^{\circledR}$ (Genomic Health, Inc., CA, USA) score and a good proliferation response to a 3-week preoperative endocrine therapy [101].

The use of carboplatin area under the curve 1.5 weekly in addition to an anthracycline-taxane containing neoadjuvant therapy improved the pathological complete remission (pCR) rate (46.7 vs $37.2 \%$ ) in the GBG-AGO-B Geparsixto-trial, particularly in triple-negative tumors ( $\mathrm{pCR}$ rate 58.7 vs $37.9 \%)$. However, it was associated with increased toxicity [9].

In HER2-positive disease, the Alliance C40601 trial showed that dual blockade (trastuzumab plus lapatinib) with a backbone of 16 weeks of paclitaxel, improved pCR compared with single blockade trastuzumab versus lapatinib numerically, although not statistically (pCR breast: 56 vs 46 vs 37\%, respectively) [10].
The ACOSOG Z1041 Phase III trial did not see a difference in $\mathrm{pCR}$ rates after neoadjuvant four-times fluorouracil, epirubicin and cyclophosphamide - 12-times paclitaxel depending on whether trastuzumab was already started with the anthracycline or only with the taxane (in breast pCR: 56.5 vs $54.2 \%$, respectively; $\mathrm{p}=0.72)$ [11]. These data do not support routine use of trastuzumab concurrent with anthracyclines in the neoadjuvant setting. Last but not least, survival analysis of the NOAH trial confirmed the significant benefit from neoadjuvant trastuzumab (event-free survival [EFS] HR: 0.64; $\mathrm{p}=0.016$; OS HR: 0.66; $\mathrm{p}=0.055$ ). Interestingly, EFS in HER2-positive disease without trastuzumab was worse than that of HER2-negative disease (62 out of 118 vs 45 out of 99). pCR under trastuzumab was significantly correlated with improved EFS (HR: $0.17 ; \mathrm{p}<0.0001)$. Cumulative congestive heart failure grade III rate was $2.1 \%$ after 5 years in the trastuzumab arm [12].

In endocrine-sensitive disease, the ATTom trial $(n=6953)$ confirmed the advantage of 10 versus 5 years of tamoxifen (response rate [RR]: 0.85; $\mathrm{p}=0.003$ ). As seen in the ATLAS trial, the benefit in relapse-free survival was particularly visible after the first 5 years and continued to persist for more than 15 years. The decrease in breast cancer-related deaths was visible after about year 7 (RR: 0.88; $\mathrm{p}=0.06$ ) and amounted to an approximately $3 \%$ absolute benefit at year 15. Prolonged tamoxifen therapy was associated with an increase in endometrial cancers (102 vs $45)$ and related deaths (37 vs 20 ) with an absolute risk of approximately $0.5 \%$. Taken together, the ATLAS and aTTom trials $(n=17,477)$ demonstrate a significant benefit regarding breast cancer-related deaths (RR: 0.85; p = 0.001) and overall mortality (RR: $0.91 ; \mathrm{p}=0.008$ ) for 10 versus 5 years of tamoxifen [13]. Current AGO guidelines already recommend $5-10$ years (depending on risk and tolerability) of endocrine therapy in early breast cancer with tamoxifen in premenopausal and a sequence of tamoxifen and aromatase inhibitors in postmenopausal patients [102].

\section{Therapy in metastatic breast cancer}

In primary breast cancer, uPA and PAI-1 are ASCO-recommended prognostic biomarkers for node-negative disease. Recent 10-year longterm follow-up of the prospective Chemo N0 trial validated these prognostic markers at highest level of evidence according to the criteria by [14] and demonstrated that uPA/PAI-1 high-risk 
patients benefit substantially from adjuvant chemotherapy [15]. For the first time, an oral uPA inhibitor, WX 671 (upamostat), was evaluated in breast cancer. In the first-line setting, there was no significant benefit from adding WX671 to capecitabine in an unselected patient population. In the exploratory analysis, patients with prior adjuvant chemotherapy seemed to benefit with a median PFS of 8.3 versus 4.3 months. There were no toxicities beyond those expected for capecitabine [16]. At ASCO 2010, WX671 was associated with prolonged survival in pancreatic cancer when combined with gemcitabine chemotherapy [17]. Further evaluation of the anti-uPA therapy - preferably in a biomarker-guided trial concept - seems promising.

The highlight of the metastatic breast cancer sessions was the first presentation of the BOLERO-3 results regarding addition of everolimus to trastuzumab and vinorelbine after trastuzumab failure. The study met its primary end point by improving median PFS from 5.78 to 7 months (HR: $0.78 ; \mathrm{p}=0.0067$ ) by adding $5 \mathrm{mg} / \mathrm{day}$ of everolimus. This was associated, however, with an increase in toxicities such as stomatitis, rash, diarrhea and fatigue but not a decrease in quality of life compared with the control arm [18]. BOLERO-3 demonstrates for the first time that mTOR inhibition may overcome trastuzumab resistance. However, considering the small PFS benefit and the added toxicity, the actual clinical impact of this therapy concept will still need to be determined, in particular once T-DM1 becomes available internationally. Nevertheless, the fact that the number of effective anti-HER 2 therapy options is constantly increasing is good news for our patients.

\section{Conclusion}

Similar to last year, this year's ASCO confirmed a number of therapy concepts in breast cancer without immediately requiring changes to our current guidelines or everyday clinical practice. Another highlight of the breast cancer sessions was the David A Karnofsky Lecture, which is associated with ASCO'S highest clinical award and this year was held by Martine Piccart (Breast International Group; Brussels, Belgium). Piccart, who was honored for her international trial network, Breast International Group, described the past and future of academic clinical trials and the resulting impact on breast cancer management in her visionary talk. The Karnofsky lecture, together with all the other interesting talks and posters can be seen in the ASCO Virtual Meeting [103].

\section{Acknowledgements \\ $N$ Harbeck is an ambassador for Oncology Central. \\ Financial \& competing interests disclosure \\ $N$ Harbeck has received honoraria for consulting and lectures from Roche and Novartis. The author has no other relevant affiliations or financial involvement with any organization or entity with a financial interest in or financial conflict with the subject matter or mate- rials discussed in the manuscript apart from those disclosed. \\ No writing assistance was utilized in the production of this manuscript.}

\section{References}

1. Shastri SS, Mittra I, Mishra G, Gupta S, Dikshit R, Badwe RA. Effect of visual inspection with acetic acid (VIA) screening by primary health workers on cervical cancer mortality: a cluster randomized controlled trial in Mumbai, India. J. Clin. Oncol. 31(Suppl.), Abstract 2 (2013).

2. Tewari KS, Sill M, Long HJ et al. Incorporation of bevacizumab in the treatment of recurrent and metastatic cervical cancer: a Phase III randomized trial of the Gynecologic Oncology Group. J. Clin. Oncol. 31(Suppl.), Abstract 3 (2013).

3. Du Bois A, Floquet A, Kim JW et al. Randomized, double-blind, Phase III trial of pazopanib versus placebo in women who have not progressed after first-line chemotherapy for advanced epithelial ovarian, fallopian tube, or primary peritoneal cancer (AEOC): results of an international Intergroup trial
(AGO-OVAR16). J. Clin. Oncol. 31(Suppl.), Abstract LBA5503 (2013).

4. Perren TJ, Swart AM, Pfisterer J et al.; ICON7 Investigators. A Phase 3 trial of bevacizumab in ovarian cancer. $N$. Engl. J. Med. 365(26), 2484-2496 (2011).

5. Burger RA, Brady MF, Bookman MA et al.; Gynecologic Oncology Group. Incorporation of bevacizumab in the primary treatment of ovarian cancer. N. Engl. J. Med. 365(26), 2473-2483 (2011).

6. Julian TB, Anderson SJ, Krag DN et al. 10-yr follow-up results of NSABP B-32, a randomized Phase III clinical trial to compare sentinel node resection (SNR) to conventional axillary dissection (AD) in clinically node-negative breast cancer patients. J. Clin. Oncol. 31(Suppl.), Abstract 1000 (2013).

7. Rutgers EJ, Donker M, Straver ME et al. Radiotherapy or surgery of the axilla after a positive sentinel node in breast cancer patients: final analysis of the EORTC AMAROS trial (10981/22023). J. Clin. Oncol. 31(Suppl.), Abstract LBA1001 (2013).

8. Gnant M, Dowsett M, Filipits M et al. Identifying clinically relevant prognostic subgroups in node-positive postmenopausal $\mathrm{HR}+$ early breast cancer patients treated with endocrine therapy: a combined analysis of 2,485 patients from ABCSG-8 and ATAC using the PAM50 risk of recurrence (ROR) score and intrinsic subtype. J. Clin. Oncol. 31(Suppl.), Abstract 506 (2013).

9. Von Minckwitz G, Schneeweiss A, Salat C et al. A randomized Phase II trial investigating the addition of carboplatin to neoadjuvant therapy for triple-negative and HER2-positive early breast cancer (GeparSixto). J. Clin. Oncol.31(Suppl.), Abstract 1004 (2013).

10. Carey LA, Berry DA, Ollila D et al. Clinical and translational results of CALGB 40601: 
a neoadjuvant Phase III trial of weekly paclitaxel and trastuzumab with or without lapatinib for HER2-positive breast cancer. J. Clin. Oncol. 31(Suppl.), Abstract 500 (2013).

11. Buzdar A, Suman VJ, Meric-Bernstam F et al. ACOSOG Z1041 (Alliance): definitive analysis of randomized neoadjuvant trial comparing FEC followed by paclitaxel plus trastuzumab $(\mathrm{FEC} \rightarrow \mathrm{P}+\mathrm{T})$ with paclitaxel plus trastuzumab followed by FEC plus trastuzumab $(\mathrm{P}+\mathrm{T} \rightarrow \mathrm{FEC}+\mathrm{T})$ in HER $2^{+}$ operable breast cancer. J. Clin. Oncol. 31(Suppl.), Abstract 502 (2013).

12. Gianni L, Eiermann W, Semiglazov V et al. Follow-up results of NOAH, a randomized Phase III trial evaluating neoadjuvant chemotherapy with trastuzumab $(\mathrm{CT}+\mathrm{H})$ followed by adjuvant $\mathrm{H}$ versus $\mathrm{CT}$ alone, in patients with HER2-positive locally advanced breast cancer. J. Clin. Oncol. 31(Suppl.), Abstract 503 (2013).

13. Gray RG, Rea D, Handley K et al. aTTom: Long-term effects of continuing adjuvant tamoxifen to 10 years versus stopping at 5 years in 6,953 women with early breast cancer. J. Clin. Oncol. 31(Suppl.), Abstract 5 (2013).

14. Hayes DF, Bast RC, Desch CE et al. Tumor marker utility grading system: a framework to evaluate clinical utility of tumor markers. J. Natl Cancer Inst. 88(20), 1456-1466 (1996).

15. Harbeck N, Schmitt M, Meisner C et al. Ten-year analysis of the prospective multicentre Chemo-N0 trial validates American Society of Clinical Oncology (ASCO)-recommended biomarkers uPA and PAI-1 for therapy decision making in node-negative breast cancer patients. Eur. J. Cancer 49(8), 1825-1835 (2013).

16. Goldstein LG, Oliveira CT, Heinrich B et al. A randomized double-blind Phase II study of the combination of oral WX-671 plus capecitabine versus capecitabine monotherapy in first-line HER2-negative metastatic breast cancer (MBC). J. Clin. Oncol. 31(Suppl.), Abstract 508 (2013).

17. Heinemann V, Ebert MP, Laubender RP, Bevan P, Mala C, Boeck S. Phase II randomised proof-of-concept study of the urokinase inhibitor upamostat (WX-671) in combination with gemcitabine compared with gemcitabine alone in patients with non-resectable, locally advanced pancreatic cancer. Br. J. Cancer 108(4), 766-770 (2013).

18. O’Regan R, Ozguroglu M, Andre F et al. Phase III, randomized, double-blind, placebo-controlled multicenter trial of daily everolimus plus weekly trastuzumab and vinorelbine in trastuzumab-resistant, advanced breast cancer (BOLERO-3). J. Clin. Oncol. 31(Suppl.), Abstract 505 (2013).

\section{Websites}

101. Women's Healthcare Study Group. www.wsg-online.com

102. AGO Breast Cancer Guidelines 2013. Recommendations for diagnosis and therapy of primary and metastatic breast cancer. www.ago-online.de

103. American Society of Clinical Oncology. Annual 2013 Meeting. http://chicago2013.asco.org/ 